\title{
Cerebrovascular prophylaxis of the frail elderly in the rehabilitation setting: a challenge for the geriatrician
}

\begin{abstract}
The rehabilitation setting is very often found to manage patients suffering from diseases of internal and geriatric interest, as well as orthopedic and neurological ones. The choice, for example, of thromboembolic prophylaxis and of the most suitable and safe drugs in these patients often requires that a physician with geriatric skills be present alongside the physiatrist. Sometimes it is also difficult for the geriatrician to assess patients, sometimes even very complex, and for this reason the presence of a Geriatrician in Rehabilitation is an added value for the care team as well as an exciting challenge for those who take care of patients frail elderly.
\end{abstract}

Keywords: rehabilitation, cerebrovascular prevention, anticoagulation therapy, elderly patient, echocardiography; stroke; atrial fibrillation
Volume 4 Issue 3 - 2019

\author{
Valerio Massimo Magro,' Carla Coppola, ${ }^{2}$ \\ Michele Caturano, ${ }^{3}$ Walter Verrusio ${ }^{4}$ \\ 'Echocardiography unit. Department of Internal Medicine and \\ Geriatry, Second University of Naples, Italy \\ ${ }^{2}$ Department of Internal Medicine, AORN San Giovanni di Dio e \\ Ruggi d'Aragona, Italy \\ ${ }^{3}$ Department of Cardiovascular, Respiratory, Nephrological, \\ Anesthesiological and Geriatric Sciences, Sapienza University of \\ Rome, Italy \\ ${ }^{4}$ Department of Intensive Rehabilitation, Casa di Cura Alma \\ Mater SpA, Italy
}

Correspondence: Valerio Massimo Magro, Department of Internal Medicine and Geriatry, University of Campania Luigi Vanvitelli, Piazza L. Miraglia 2, 80100 , Naples, Italy, Tel 3492224922, Email valerio_magro@hotmail.com

Received: February 17, 2019 | Published: May 15, 2019

\section{Background}

The prophylaxis of thromboembolic events plays an important part of the patient's treatment of atrial fibrillation and is also an important reason for treatment of patients who have experienced a stroke with disabling outcomes. Anticoagulant treatment has both benefits and risks for elderly patients. Benefits include the prevention of ischemic and thromboembolic events and this action is important in a rehabilitation setting, where the patient arrives with motor deficits and periods of variable duration to be spent between the bed and the rehabilitation sessions. The choice to undertake an anticoagulant treatment in rehabilitation setting appears crucial because the adverse events due to both the therapy and the patient's status (frailty, tendency to fall) can undermine the success of the rehabilitation itself or be - at least potentially - due to new exacerbations.

\section{Case report}

We observed a 73-year-old female patient with hypertension and atrial fibrillation (AF) with an unspecified onset (already treated with apixaban). the patient was still hit by stroke about a week before our care. The drug therapy he presented to us was digital therapy with a dose of $0.125 \mathrm{mg}$, enalapril/lercadipine $20 / 20 \mathrm{mg}$, atorvastatin $80 \mathrm{mg}$. On the EKG, sinus rhythm demonstrated a deviated left axis, HR 75 bpm, left branch block, and intermittent anti-hypertensive therapy for episodes of symptomatic hypotension, The patient was taking these medications at the time: acetylsalicylic acid (ASA) $100 \mathrm{mg}$, Torvast $40 \mathrm{mg}$, Optovitreo ${ }^{\circledR}$ (Figure 1). On our physical examination, the patient appears in a slight deteriorated general medical condition. We noted to semeiologic maneuvers a objectivity characterized by a deficit of strength in the left limbs with positive Mingazzini sign. We also required laboratory tests and a radiological assessment: blood tests within limits except creatinine $1.3 \mathrm{mg} / \mathrm{dl}$, serum sodium concentration $134 \mathrm{mEq} / \mathrm{l}$, total cholesterol $262 \mathrm{mg} / \mathrm{dl}$, LDL-C 180 $\mathrm{mg} / \mathrm{dl}$. Negativity of the cardiomarkers curve due to acute changes. Presence of a hypodense areola with a nucleus-capsular center on the right at the brain computed tomography $(\mathrm{CT})$ imaging. There were no significant stenoses to the carotid arteries at the ultrasound evaluation, while at the echocardiography there was a reduced ejection fraction (EF), dyskinesia of the interventricular septum (SIV) with a left atrial enlargement and a mitro-tricuspid insufficiency. For these reasons, we modified the therapy by adding cautiously and with weighted timing rivaroxaban $10 \mathrm{mg}$, ASA $100 \mathrm{mg}$, reserving a re-evaluation of the lipid profile at a greater distance of time before changing the lipidlowering therapy. A subsequent brain $\mathrm{CT}$ scan showed no bleeding in progress, with the capsular lesion remaining unchanged. A careful physiatric (Funtional Independence Measure, FIM) (Figure 2) and geriatric assessment (multidimensional assessment and evaluation of the risk of falls, utilization of ATRIA, HAS-BLED and ORBIT scores) (Figure 3) was performed; therefore active physiotherapy was provided and motor coordination exercises of the four limbs, station control erect, postural passages and exercises for the correct recovery of the gait scheme. Normal medical follow-up during hospitalization and home discharge after 2 months with unchanged therapy. 


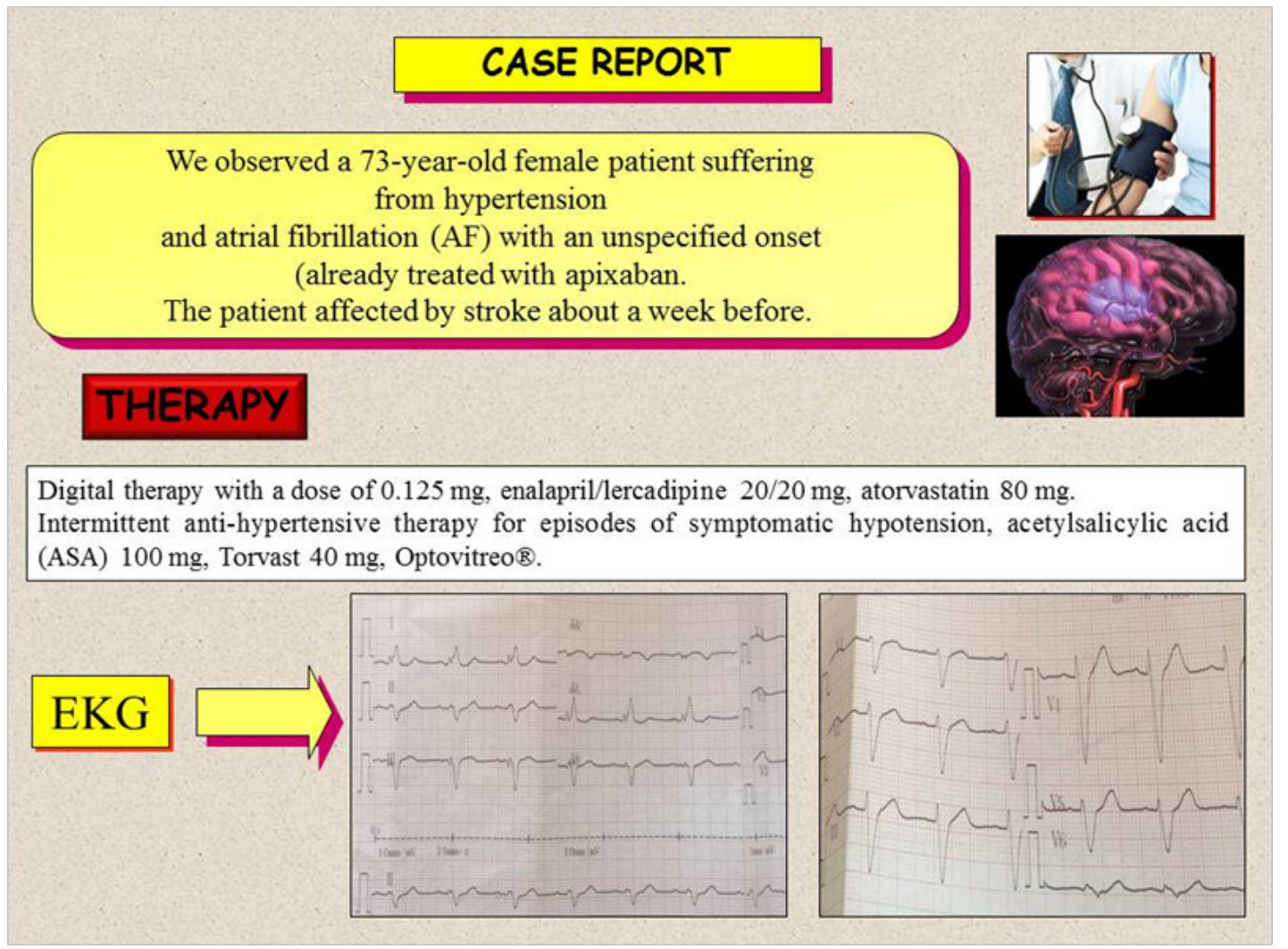

Figure I The figure shows both the patient's remote and proximate pathological anamnesis, highlighting the recent cerebrovascular episode that occurred under therapy with new anticoagulant drug, both the therapy and the electrocardiographic pattern, which shows a left bundle branch block.

\begin{tabular}{|c|c|c|c|}
\hline & ADMISSION & DISCHARGE & FOLLOW-UP \\
\hline $\begin{array}{l}\text { Self-Care } \\
\text { A. Eating } \\
\text { B. Grooming } \\
\text { C. Bathing } \\
\text { D. Dressing - Upper Body } \\
\text { E. Dressing - Lower Body } \\
\text { F. Toileting }\end{array}$ & & & \\
\hline $\begin{array}{l}\text { Sphincter Control } \\
\text { G. Bladder Management } \\
\text { H. Bowel Management }\end{array}$ & & & \\
\hline $\begin{array}{l}\text { Transfers } \\
\text { I. Bed, Chair, Wheelchair } \\
\text { J. Toilet } \\
\text { K. Tub, Shower } \\
\text { Locomotion }\end{array}$ & & & \\
\hline $\begin{array}{l}\text { L. Walk/Wheelchair } \\
\text { M. Stairs } \\
\text { Motor SubtotalScore }\end{array}$ & & & \\
\hline $\begin{array}{l}\text { Communication } \\
\text { N. Comprehension } \\
\text { O. Expression } \\
\text { Social Cognition }\end{array}$ & & & \\
\hline $\begin{array}{l}\text { P. Social Interaction } \\
\text { Q. Problem Solving } \\
\text { R. Memory } \\
\text { Cognitive Subtotal Score } \\
\text { TOTAL FIM Score }\end{array}$ & & & \\
\hline
\end{tabular}

Figure 2 The FIM (Funtional Independence Measure) is the scale used in the physiatric field to evaluate the functional disability of patients at the entrance, at the exit and to monitor the progress of the recovery during rehabilitation therapy. 


\section{HAS-BLED}

\begin{tabular}{|c|c|c|}
\hline Letter & Clinical Characteristic & Points \\
\hline H & Hypertension & 1 \\
\hline A & Abnormal Liver or Renal Function & 1 or 2 \\
\hline S & Stroke & 1 \\
\hline B & Bleeding & 1 \\
\hline L & Labile INR & 1 \\
\hline E & Elderly (age $>65)$ & 1 \\
\hline D & Drugs or Alcohol & 1 or 2 \\
\hline Maximum Score & & 9 \\
\hline
\end{tabular}

Figures 3 The HAS-BLED is a scoring system developed to assess I-year risk of major bleeding in patients taking anticoagulants with atrial fibrillation. It was developed in 2010 with data from 3,978 patients in the Euro Heart Survey.

\section{Discussion}

Prophylaxis of acute cerebrovascular events in elderly patients remains a topic of extreme interest, as timing and the choice of suitable drugs can have important repercussions on the prognosis of these individuals. The wide range of drugs available today, as well as the increasing comorbidity of patients affected by stroke has made it difficult to take charge. Here we discuss the risks and benefits of thrombo-embolic prophylactic treatment in the affected patient while undergoing rehabilitative treatment, by analyzing this clinical case. A recent study that evaluated a matched cohort study in patients treated with vitamin K antagonists at a thrombosis service, Jan2009-Jun 2012, observed the same risk (risk of bleeding; major bleeding and risk of thrombosis) in 1104 patients in our patient's age range. ${ }^{1}$ Although the guidelines of the European Society of Cardiology (ESC) recommend the use of the CHADS VASC score for the definition of thrombotic risk, ${ }^{2,3}$ we have used the ATRIA score for its greater discriminatory capacity, as described in the literature, together with the ORBIT. More recent studies, however, question this concept and, while the use of certain scores in place of others (and only those) is advocated in certain guidelines (ESC) rather than in others, the study by Yao $\mathrm{X}$ et $\mathrm{al},{ }^{5}$ comparing the different scores for continuous variables (major and intracranial bleeding), did not find great differences in sensitivity curves. In conclusion, CHA2DS2-VASC, CHADS2, HASBLED, ORBIT and ATRIA had similar, albeit modest, performance in predicting non-vitamin $\mathrm{K}$ antagonist oral anticoagulants (NOAC)- associated bleeding in patients with AF. Careful assessment and active management of bleeding risk factors may be warranted in all patients on NOAC who have high stroke risk scores. Moreover, the scales for haemorrhagic risk usually used have a low discriminatory capacity, especially in the elderly and the topic about the plasma concentrations of NAOs remains open and a source of discussion in the scientific community. A recent Italian position paper provides clinicians with recommendations useful in managing some important issues regarding the use of anticoagulant therapy in patients with $\mathrm{AF}$ in particularly complex clinical situations, such as our case study and other, patients with comorbidities. ${ }^{6}$ At the same time, the role of anticoagulation therapy in patients who have already sustained brain damage lacks specific evidence and needs further investigation. In particular, the safety and efficacy of restarting anticoagulation therapy after an acute, ischemic or hemorrhagic cerebrovascular event remain unclear. ${ }^{7,8}$ Hemorrhagic events are a common clinical problem in the elderly, and pose difficult decision-making. Studies suggest that the recovery of oral anticoagulant therapy after hemorrhagic event (both in the gastrointestinal tract but also in another location, such as a cerebral or abdominal localization) is associated with better outcome clinics (lower mortality and lower thromboembolic events) but with a possible increase in the risk of general hemorrhagic recurrences and gastrointestinal bleeding. However, this evidence primary comes from observational registers, mostly retrospective, as opposed to global patient assessments. Thus, these date have not been subjected to statistical analysis, carry a high risk of bias, and should be considered 
with caution. ${ }^{9}$ Our patient had a cerebrovascular event despite taking Apixaban. Doubts about the cost effectiveness ratio of the molecule had arisen in older patients and those at high risk of adverse events. ${ }^{10,11}$ On the other hand, the consensus of experts from the American College of Cardiology (ACC) considers these patients to be at high thrombotic risk and encourages anticoagulant therapy ${ }^{12}$ while the councils of the ESC and others more often concern patients who have experienced bleeding episodes rather than those who experienced ischemia during therapy ${ }^{13,14}$ In the choice of therapy, despite the doubts still existing on thromboembolic prophylaxis in tertiary prevention (stroke already occurred), ${ }^{15}$ we have adhered to the recommendations of the Stroke Prevention and Educational Awareness Diffusion (SPREAD) Italian guidelines of Italian Stroke Organization (ISO) (which, in patients with a higher risk of intracranial bleeding, suggest this behavior with NOAC for the best safety profile), ${ }^{16}$ recent results of both the COMPASS trial (which studied Rivaroxaban also in cardiovascular prevention) ${ }^{17}$ and studies such as XANTUS and REVISIT-US, ${ }^{18-20}$ which evaluated the safety on stroke prevention and on the incidence of intracranial bleeding compared a Warfarin. In addition, the patient had a left bundle branch block and in a rehabilitation setting there may not be the possibility of verifying whether the electrocardiographic anomaly is of new onset (absence of previous traces). The real life data reconfirms the positive risk/benefit profile of rivaroxaban in patients with non-valvular AF and confirms the efficacy and safety profile of rivaroxaban, in line with those of the pivotal Phase III ROCKET AF study and of other real life studies. All this is associated with an evaluation of the individual hemorrhagic risk of the patient, both in the use of scores and in a specialized joint assessment, in a rehabilitative setting. The possibility that the patient will experience a higher fall risk once she is transferred to rehabilitation setting from the acute hospital, is not a remote possibility. In fact, the atrial fibrillation itself, the motor damage caused by the stroke, and the comorbidities, can all undermine the patient's static and dynamic balances, making it fragile. Also contributing to this fragility are changes in the body's homeostasis due to environmental stimuli (different treatment settings, rehabilitative pathways. But also postural passages and various physical needs). A fall can not only be a cause of serious adverse event for the patient in prophylactic stroke therapy (hematoma and bleeding in different districts), be due to discontinuation of therapy (with exposure of the patient to new thrombotic events), but also undermine his rehabilitation path being the cause of interruption of the same and re-admission to an acute department ${ }^{21}$ Although this risk exists, this must not be considered as an anticoagulant treatment and indeed the patient's stratification during the execution of a rehabilitation treatment must provide a careful assessment of the risk of falling through a close collaboration between geriatrician, physiatrist, physiotherapists, speech therapists and nursing staff and social and health workers. ${ }^{22,23}$ For this reason, in the evaluation of our patient we used a functional mobility scale (FIM), integrating the eminently physiatrist specialist vision with the multidimensional evaluation - eminently geriatric practice - as well valide scales for assessing gait and balance such as the Tinetti scale. In this way the patient is well stratified and, on the contrary, he chooses the type of patient who "can safely receive the anticoagulant which reduces the fall risk due to hemorrhagic injuries in stroke patients.

\section{Conclusion}

The antithrombotic and antiplatelet therapy now appears as a consolidated acquisition for ischemic patients and with AF. Furthermore, in the patient with a thromboembolic risk due to AF, the clinician is able to reconcile two different needs, namely the prevention of the cardioembolism but also the avoidance of the hemorrhagic complications. This assessment becomes crucial for elderly patients and specially in the rehabilitation settings, where each event can delay the treatment aimed at functional improvement or cause it to fail. A correct estimate of the risks and functional abilities of the patient becomes imperative in this setting. For these reasons the role of the geriatrician in the rehabilitation setting is essential.

\section{Acknowledgments}

None.

\section{Conflict of interests}

Authors declare that there is no conflict of interest

\section{References}

1. Kooistra HA, Calf AH2, Piersma-Wichers M, et al. Risk of bleeding and thrombosis in patients 70 years or older using vitamin $\mathrm{K}$ antagonists. JAMA Intern Med. 2016;176(8):1176-1183.

2. Ponikowski P, Voors AA, Anker SD, et al. 2016 ESC Guidelines for the diagnosis and treatment of acute and chronic heart failure: The Task Force for the diagnosis and treatment of acute and chronic heart failure of the European Society of Cardiology (ESC)Developed with the special contribution of the Heart Failure Association (HFA) of the ESC. Eur Heart J. 2016;37(27):2129-2200.

3. Steffel J, Verhamme P, Potpara TS, et al. The 2018 European heart rhythm association practical guide on the use of non-vitamin $\mathrm{K}$ antagonist oral anticoagulants in patients with atrial fibrillation. Eur Heart J. 2018;39(16):1330-1393.

4. Van den Ham HA, Klungel OH, Singer DE, et al. Comparative performance of ATRIA, CHADS2, and CHA2DS2-VASc risk scores predicting stroke in patients with atrial fibrillation: results from a national primary care database. J Am Coll Cardiol. 2015;66(17):1851-1859.

5. Yao X, Gersh BJ, Sangaralingham LR, et al. Comparison of the CHA2DS2-VASc, CHADS2, HAS-BLED, ORBIT, and ATRIA risk scores in predicting non-vitamin $\mathrm{k}$ antagonist oral anticoagulantsassociated bleeding in patients with atrial fibrillation. Am J Cardiol. 2017;120(9):1549-1556.

6. Botto GL, Padeletti L, Ammirati F, et al. Appropriateness criteria for the management of anticoagulant therapy in complex patients with atrial fibrillation. The opinion of a group of expert Italian cardiologists. G Ital Cardiol (Rome). 2018;19(10):591-600.

7. Murthy SB, Gupta A, Merkler AE, et al. Restarting anticoagulant therapy after intracranial hemorrhage: a systematic review and meta-analysis. Stroke. 2017;48(6):1594-1600.

8. Hankey GJ, Norrving B, Hacke W, et al. Management of acute stroke in patients taking novel oral anticoagulants. Int J Stroke. 2014;9(5):627-632.

9. Staerk L, Fosbøl EL, Lamberts M, et al. Resumption of oral anticoagulation following traumatic injury and risk of stroke and bleeding in patients with atrial fibrillation: a nationwide cohort study. Eur Heart $J$. 2018;39(19):1698-1705.

10. $\mathrm{Ng} \mathrm{KH}$, Shestakovska O, Connolly SJ, et al. Efficacy and safety of apixaban compared with aspirin in the elderly: a subgroup analysis from the AVERROES trial. Age Ageing. 2016;45(1):77-83.

11. Lee S, Anglade MW, Meng J, et al. Cost-effectiveness of apixaban compared with aspirin for stroke prevention in atrial fibrillation among patients unsuitable for warfarin. Circ Cardiovasc Qual Outcomes. 2012;5(4):472-479. 
12. Tomaselli GF, Mahaffey KW, Cuker A, et al. 2017 ACC expert consensus decision pathway on management of bleeding in patients on oral anticoagulants. J Am Coll Cardiol. 2017;70(24):3042-3067.

13. Halvorsen S, Storey RF, Rocca B, et al. Management of antithrombotic therapy after bleeding in patients with coronary artery disease and/or atria fibrillation: expert consensus paper of the european society of cardiology working group on thrombosis. Eur Heart J. 2017;38(19):1455-1462.

14. Nielsen PB, Larsen TB, Skjøth F, et al. Restarting anticoagulant treatment after intracranial hemorrhage in patients with atrial fibrillation and the impact on recurrent stroke, mortality, and bleeding: a nationwide cohort study. Circulation. 2015;132:517-525.

15. Abrignani MG, Carletti M, Bovi P, et al. Prevention of cardioembolic events after intracranial hemorrhage. G Ital Cardiol. 2018; 19(10):542551.

16. Stroke prevention and educational awareness diffusion (SPREAD) Italian guidelines. VIIIth edition; 2016

17. Bosch J, Eikelboom JW, Connolly SJ, et al. Rationale, design and baseline characteristics of participants in the cardiovascular outcomes for people using anticoagulation strategies (COMPASS) trial. Can J Cardiol. 2017;33(8):1027-1035.
18. Martínez CAA, Lanas F, Radaideh G, et al. XANTUS-EL: A real-world, prospective, observational study of patients treated with rivaroxabanfor stroke prevention in atrial fibrillation in Eastern Europe, Middle East, Africa and Latin America. Egypt Heart J. 2018;70(4):307-313.

19. Riva L, Di Pasquale G. New oral anticoagulants in patients with atrial fibrillation: efficacy and safety data from the real world. G Ital Cardiol (Rome). 2017;18(3):188-198.

20. Camm AJ, Amarenco P, Haas S, et al. XANTUS: a real-world, prospective, observational study of patients treated with rivaroxaban for stroke prevention in atrial fibrillation. European Heart Journal. 2016;37(14):1145-1153.

21. Ross MK, Egan E, Zaman M, Falls in the inpatient rehabilitation facility. Phys Med Rehabil Clin N Am. 2012;23(2):305-314.

22. Garwood CL, Corbett TL. Use of anticoagulation in elderly patients with atrial fibrillation who are at risk for falls. Ann Pharmacother. 2008;42(4):523-532.

23. Stein J, Viramontes BE, Kerrigan DC. Fall-related injuries in anticoagulated stroke patients during inpatient rehabilitation. Arch Phys Med Rehabil. 1995;76(9):840-843. 